\title{
IS AN IRNG SINGLY GENERATED AS AN IDEAL?
}

\author{
NICOLAS MONOD, NARUTAKA OZAWA, AND ANDREAS THOM
}

\begin{abstract}
Recall that a rng is a ring which is possibly non-unital. In this note, we address the problem whether every finitely generated idempotent rng (abbreviated as irng) is singly generated as an ideal. It is well-known that it is the case for a commutative irng. We prove here it is also the case for a free rng on finitely many idempotents and for a finite irng. A relation to the Wiegold problem for perfect groups is discussed.
\end{abstract}

\section{INTRODUCTION}

The Wiegold problem (FP14 in [1]) is a longstanding problem in group theory. It asks whether every finitely generated perfect group $G$ (i.e., $G=[G, G]$ ) is singly generated as a normal subgroup. (We note that it makes no difference if one replaces "finitely generated" with "finitely presented" in the statement.) Wiegold answered it positively in the finite group case, see [3, 4.2] for a stronger fact. In this paper, we address a similar problem for rngs. Recall that a rng (a.k.a. a pseudo-ring) is an algebraic structure satisfying the same properties as a ring, except that it may not have a multiplicative unit. Every rng $R$ is an ideal of a ring, say, the unitization $R^{+}$of $R$. We call a rng $R$ an irng (a shorthand for an idempotent rng) if it satisfies $R^{2}=R$. Here $R^{2}=\operatorname{span}\{x y: x, y \in R\}$. The weight of a $\operatorname{rng} R$ is defined as

$$
w(R)=\min \{n: R \text { is generated by } n \text { elements as an ideal }\} .
$$

Obviously any unital rng is an irng with weight one. We also notice that $w(R)$ is at least the minimal number of generators of the additive group $R / R^{2}$. The direct sum $R=\bigoplus \mathbf{Z}$ of infinitely many copies of $\mathbf{Z}$ is an example of irng with $w(R)=+\infty$, but not finitely generated. In this paper, we address the following.

Problem. Find a finitely generated irng whose weight is larger than one; more generally, whose weight is larger than any prescribed positive integer.

Even without the finite generation assumption, there seems no known example of irng $R$ with $1<w(R)<+\infty$. For a subset $Z$ in a rng $R$, we denote by $\langle\langle Z\rangle\rangle$ the ideal generated

2000 Mathematics Subject Classification. 16A99; 20F05.

Key words and phrases. Wiegold problem, idempotent rng, irng.

N.M. was partially supported by the SNF and the ERC. N.O. was partially supported by JSPS and Sumitomo Foundation. A.T. was supported by the ERC Starting Grant 277728. 
by $Z$. We note that if an irng $R$ satisfies $R=\langle\langle Z\rangle\rangle$, i.e., $R=\operatorname{span}(Z+R Z+Z R+R Z R)$, then because $R^{3}=R$ it actually satisfies $R=\operatorname{span} R Z R$.

\section{IRNGS WITH WEIGHT ONE}

The following result is well-known, but we include the proof for the reader's convenience. See Theorem 76 in [2] for more general fact.

Theorem 1. Every finitely generated commutative irng $R$ has a unit and hence $w(R)=1$.

Proof. Let $x_{1}, \ldots, x_{n}$ be generators and $x=\left[x_{1}, \ldots, x_{n}\right]^{T} \in M_{n, 1}(R)$. Since $R^{2}=R$, there is $A \in M_{n}(R)$ such that $A \boldsymbol{x}=\boldsymbol{x}$. Let $d$ be the determinant of $I-A$, considered in the unitization $R^{+}$. Then, $z=1-d \in R$. By Cramer's formula, one has

$$
\operatorname{diag}(d, \ldots, d) \boldsymbol{x}=(I-A)^{\sim}(I-A) \boldsymbol{x}=\mathbf{0},
$$

where $(I-A)^{\sim}$ is the adjugate matrix (the transpose of the matrix of cofactors). This means that $z x_{i}=x_{i}$ for all $i$, and $z$ is a unit for $R$.

Theorem 2. Let $R$ be a rng generated by $\left\{x_{1}, \ldots, x_{n}\right\}$ as an ideal and assume that for every $i$ there is $u_{i} \in\left\langle\left\langle x_{i}, \ldots, x_{n}\right\rangle\right\rangle$ such that either $x_{i}=x_{i} u_{i}$ or $x_{i}=u_{i} x_{i}$. Then, $w(R)=1$.

Proof. Define elements $w_{1}, \ldots, w_{n}$ in $1+R \subseteq R^{+}$by setting $w_{1}=1-u_{1}$ and for $i \geq 2$

$$
w_{i}= \begin{cases}w_{i-1}\left(1-u_{i}\right) & \text { if } x_{i}=u_{i} x_{i} \\ \left(1-u_{i}\right) w_{i-1} & \text { if } x_{i}=x_{i} u_{i}\end{cases}
$$

Let further $z_{i}=1-w_{i} \in R$; we claim that $R=\left\langle\left\langle z_{n}\right\rangle\right\rangle$.

Notice first that for all $i$ we have $x_{i}=z_{i} x_{i}$ or $x_{i}=x_{i} z_{i}$; in any case, $x_{i} \in\left\langle\left\langle z_{i}\right\rangle\right\rangle$. It follows that $u_{i} \in\left\langle\left\langle z_{i}, \ldots, z_{n}\right\rangle\right\rangle$. Since $z_{i}-z_{i-1}$ simplifies (for $i \geq 2$ ) to $u_{i} w_{i-1}$ or to $w_{i-1} u_{i}$, we further deduce that $z_{i-1}$ is in $\left\langle\left\langle z_{i}, \ldots, z_{n}\right\rangle\right\rangle$. Thus,

$$
\left\langle\left\langle z_{n}\right\rangle\right\rangle=\left\langle\left\langle z_{1}, \ldots, z_{n}\right\rangle\right\rangle=\left\langle\left\langle x_{1}, \ldots, x_{n}\right\rangle\right\rangle=R
$$

as claimed.

We have the particular case mentionned in the abstract by setting $u_{i}=x_{i}$ :

Corollary 3. The free rng on finitely many idempotents is singly generated as an ideal.

We state and prove two further corollaries of Theorem 2 ,

Corollary 4. Every finite irng $R$ is generated by idempotents as an ideal and $w(R)=1$.

Proof. Let $R$ be a finite irng and $I$ be the ideal generated by the idempotents in $R$. Suppose by contradiction that $I \neq R$. Then, $R / I$ is a finite irng and hence contains a non-nilpotent element (e.g., by Levitzki's theorem). Let $a \in R$ be a pre-image of a nonnilpotent element in $R / I$. Since $R$ is finite, $a^{m}=a^{n}$ for some $m \neq n$. Since $a^{m^{k}}=a^{n^{k}}$ 
for every $k$, we may assume that $a^{m}=a^{n}$ with $m>2 n$. Then, $a^{m-n}$ is an idempotent in $R \backslash I$. A contradiction. This proves the first half, and the second half follows from the previous theorem.

Remark. We note that a finite irng need not be unital; e.g., $R=\left(\begin{array}{ccc}* & * & * \\ 0 & 0 & * \\ 0 & 0 & *\end{array}\right) \subset M_{3}(\mathbf{Z} / 2 \mathbf{Z})$.

Smoktunowicz and later Bergman have independently informed us about the following corollary of Theorem 2. With the kind permission of George Bergman we include his argument. Recall that a semigroup $S$ is called idempotent if $S^{2}=S$. We do not assume that a semigroup contains an identity element.

Corollary 5. Let $S$ be an idempotent semigroup which is finitely generated as a semigroup, or more generally, as a left ideal, and let $k$ be a unital commutative ring. The semigroup algebra $R=k S$ is generated as a 2-sided ideal by a single element.

Note that $S$ is idempotent and generated by a finite set $X \subset S$ as a left ideal if and only if $S=S X$. For the proof we need the following lemma.

Lemma 6. Let $S$ be a semigroup containing a finite set $X$ such that $S=S X$. Then $S$ is generated as a 2-sided ideal by a subset $X_{0}$ of $X$ with the property that every $x \in X_{0}$ satisfies $x \in S x S x$.

Proof. First, some notation. For $x, y \in S$, we shall write $y<_{1} x$ if $y \in S x$. Clearly, the binary relation $<_{1}$ is transitive. Thus, our assumption says $\forall y \in S \exists x \in X: y<_{1} x$.

Hence, starting with any $y \in S$, we can find an infinite chain of elements of $X$ going upward from it with respect to $<_{1}$. Since $X$ is finite, this chain must involve a repetition; and applying transitivity both to the chain from $y$ to an element $x$ which is repeated, and to the chain from that $x$ to a repetition of $x$, we get $\forall y \in S \exists x \in X: y<_{1} x<_{1} x$. Hence, letting $X_{1}=\left\{x \in X \mid x<_{1} x\right\}$, we see that $S=S X_{1}$.

In the remainder of this proof, for convenience in writing the ideal generated by an element, we shall write $S^{+}$for the monoid obtained by adjoining an identity element to $S$. We now define a second relation: For $x, y \in S$, we shall write $y<_{0} x$ if $y \in S^{+} x S^{+} y$. Again, the binary relation $<_{0}$ is transitive. Indeed, if $y \in S^{+} x S^{+} y$ and $x \in S^{+} z S^{+} x$, then $y \in S^{+} x S^{+} y \subset S^{+}\left(S^{+} z S^{+} x\right) S^{+} y \subset S^{+} z S^{+} y$, showing that $y<_{0} x<_{0} z$ implies $y<_{0} z$.

Note that for $y \in X_{1}$, if we take an element of $z \in S$ witnessing the relation $y \in S y$, i.e. $y=z y$, then $z$ will have a right factor in $X_{1}$, i.e. $z=z^{\prime} x$ with $z^{\prime} \in S^{+}$and $x \in X_{1}$. We conclude $y \in S^{+} x S^{+} y$ for some $x \in X_{1}$ and thus $\forall y \in X_{1} \exists x \in X_{1}: y<_{0} x$.

From finiteness of $X_{1}$ and a similar argument as above, we get: $\forall y \in X_{1} \exists x \in X_{1}$ : $y<_{0} x<_{0} x$. We set $X_{0}=\left\{x \in X_{1} \mid x<_{0} x\right\}$. In particular, for every $x \in X_{0}$, we have $x \in S x S x$. Moreover, for all $x \in X_{1}, x \in S^{+} X_{0} S^{+} x \subset S^{+} X_{0} S$ and hence $X_{1} \subset S^{+} X_{0} S$. We conclude $S=S X_{1} \subset S X_{0} S$. This finishes the proof.

Proof of Corollary 5. Apply Theorem 2 to the set $X_{0}$ given by Lemma 6 . 


\section{A Relation to the Wiegold problem}

Let $R$ be a rng and $n$ be a positive integer. For $1 \leq i \neq j \leq n$ and $r \in R$, we denote by $E_{i, j}(r)$ the elementary matrix with 1's on the diagonal, $r$ in the $(i, j)$-th entry, and 0's everywhere else. Thus, $E_{i, j}(R) \in \mathrm{GL}_{n}\left(R^{+}\right)$, where $R^{+}$is again the unitization of $R$. We define $\mathrm{E}_{n}(R)$ to be the subgroup of $\mathrm{GL}_{n}\left(R^{+}\right)$generated by all the elementary matrices $E_{i, j}(r)$. They satisfy the Steinberg relations:

- $E_{i, j}(r) E_{i, j}(s)=E_{i, j}(r+s)$;

- $\left[E_{i, j}(r), E_{j, k}(s)\right]=E_{i, k}(r s)$ if $i \neq k$;

- $\left[E_{i, j}(r), E_{k, l}(s)\right]=1$ if $i \neq l$ and $j \neq k$.

Recall that the weight of a group $G$ is defined as

$$
w(G)=\min \{n: G \text { is generated by } n \text { elements as a normal subgroup }\} .
$$

The following theorem relates the Wiegold problem to the irng problem.

Theorem 7. Let $R$ be a finitely generated irng and $n \geq 3$. Then $\mathrm{E}_{n}(R)$ is a finitely generated perfect group such that $w(R) / n^{2} \leq w\left(\mathrm{E}_{n}(R)\right) \leq\left\lceil 2 w(R) /\left(n^{2}-n-2\right)\right\rceil$.

Proof. That $\mathrm{E}_{n}(R)$ is finitely generated and perfect follows from the Steinberg relations. First suppose that $\mathrm{E}_{n}(R)$ is generated by $A_{1}, \ldots, A_{m}$ as a normal subgroup. Then, for the unit matrix $I$, one has $A_{i}-I \in M_{n}(R)$. Thus, the collection $Z$ of all entries of $A_{i}-I, i=1, \ldots, m$ is a subset of $R$ whose cardinality is at most $m n^{2}$. Since all $A_{i}$ 's are killed by the canonical homomorphism from $\mathrm{E}_{n}(R)$ onto $\mathrm{E}_{n}(R /\langle\langle Z\rangle\rangle)$, one sees that $R=\langle\langle Z\rangle\rangle$. This proves $w(R) \leq w\left(\mathrm{E}_{n}(R)\right) n^{2}$. To prove the other inequality, consider the upper triangular matrix $A=\left[a_{i, j}\right] \in \mathrm{E}_{n}(R)$ with 1 's on the diagonal and $a_{i, j}=0$ for $i>j$ and for $(i, j)=(1, n)$. Thus $A$ can have $\left(n^{2}-n-2\right) / 2$ many non-zero entries from $R$. For every $1 \leq i, j<n$ and $s, t \in R$, one has

$$
\langle\langle A\rangle\rangle \ni\left[E_{2, i}(s),\left[A, E_{j, n}(t)\right]\right]=\left[E_{2, i}(s), \prod_{k=1}^{n} E_{k, n}\left(a_{k, j} t\right)\right]=E_{2, n}\left(s a_{i, j} t\right) .
$$

Similarly, $E_{n, 2}\left(s a_{i, j} t\right) \in\langle\langle A\rangle\rangle$ for every $1<i, j \leq n$ and $s, t \in R$. Thus by the Steinberg relations and the fact that $R^{2}=R$, one has $E_{n}(R Z R) \subset\langle\langle A\rangle\rangle$ for $Z=\left\{a_{i, j}\right\}$. This completes the proof.

Hence, one would solve the Wiegold problem if one finds a finitely generated irng $R$ with $w(R)>9$. In this regard, the irng problem is harder than the Wiegold problem, but the authors feel that rngs may be more tractable than groups.

There is another connection to the Wiegold problem. Let $G$ be a group, $k$ be a finitely generated unital ring, and let $\omega_{k} G \subset k G$ be the augmentation ideal inside the group ring of $G$ with coefficients in $k$. Since $\omega_{k}(G) / \omega_{k}^{2}(G)=k \otimes_{\mathbf{Z}} G_{a b}, \omega_{k}(G)$ is a finitely generated irng in many cases, e.g. if $G$ is perfect. It is also clear that $w\left(\omega_{k}(G)\right) \leq w(G)$. Note that this inequality can be strict. Indeed, Theorem 2 shows that $w\left(\omega_{\mathbf{Z} / 3 \mathbf{Z}}(\mathbf{Z} / 2 \mathbf{Z} * \cdots * \mathbf{Z} / 2 \mathbf{Z})\right)=1$ 
since $\omega_{\mathbf{Z} / 3 \mathbf{Z}}(\mathbf{Z} / 2 \mathbf{Z} * \cdots * \mathbf{Z} / 2 \mathbf{Z})$ is generated by finitely many idempotents. On the other side $w(\mathbf{Z} / 2 \mathbf{Z} * \cdots * \mathbf{Z} / 2 \mathbf{Z})$ equals the number of factors in the free product. We do not know of any example where the inequality is strict if $R=\mathbf{Z}$.

It is natural to ask whether a finitely generated irng must have few generators as a left ideal in itself. In this case, more can be said using $\ell^{2}$-invariants. Recall that $b_{1}^{(2)}(G)-$ a numerical invariant of $G$ ranging in the interval $[0, \infty]$ - denotes the first $\ell^{2}$-Betti number of $G$

Theorem 8. Let $G$ be a group. Then, $\omega_{\mathbf{Z}}(G)$ needs at least $\left\lceil b_{1}^{(2)}(G)+1\right\rceil$ generators as a left ideal in itself.

Proof. If $G$ is finite, then $b_{1}^{(2)}(G)=0$. Thus, we may assume that $G$ is infinite. By [4], Lück's dimension of the space $Z^{1}\left(G, \ell^{2} G\right)$ of 1-cocycles on $G$ with values in the Hilbert space $\ell^{2} G$ - endowed with the $\mathbf{Z} G$-module structure given by the left regular representation - is equal to $b^{(2)}(G)+1$. For more information on $\ell^{2}$-Betti numbers, Lück's dimension function and the left regular representation, see [4] and the references therein.

It is well known that $Z^{1}\left(G, \ell^{2} G\right)=\operatorname{hom}_{\mathbf{Z} G}\left(\omega_{\mathbf{Z}}(G), \ell^{2} G\right)$, where $\operatorname{hom}_{\mathbf{Z} G}$ denotes the set of left-module homomorphisms. If $S \subseteq \omega_{\mathbf{Z}}(G)$ is a set of generators of $\omega_{\mathbf{Z}}(G)$ as a left-ideal in itself. It follows that $\operatorname{hom}_{\mathbf{z} G}\left(\omega_{\mathbf{Z}}(G), \ell^{2} G\right) \subseteq \ell^{2} G^{\oplus S}$ by evaluation. Since $\operatorname{dim}_{G} \ell^{2} G^{\oplus S}=|S|$, the claim follows.

There are examples of finitely generated perfect groups with first $\ell^{2}$-Betti number as large as we wish; any free product of non-trivial perfect groups works. Thus, for any positive integer $k$, a finitely generated irng can be found which needs at least $k$ elements to generate it as a left ideal.

Acknowledgment. This research was carried out while the authors were visiting at the Institut Henri Poincaré (IHP) for the Program "von Neumann algebras and ergodic theory of group actions." The authors gratefully acknowledge the kind hospitality and stimulating environment provided by IHP and the program organizers. The authors would like to thank Professor Agata Smoktunowicz and Professor George Bergman for their help regarding the irng problem, and Professor Martin Kassabov for improving the estimate in Theorem 7 .

\section{REFERENCES}

[1] G. Baumslag, A.G. Myasnikov, V. Shpilrain; Open problems in combinatorial group theory. Second edition. Combinatorial and geometric group theory (New York, 2000/Hoboken, NJ, 2001), 1-38, Contemp. Math., 296, Amer. Math. Soc., Providence, RI, 2002.

[2] I. Kaplansky; Commutative rings. Allyn and Bacon, Inc., Boston, Mass. $1970 \mathrm{x}+180$ pp.

[3] J. C. Lennox and J. Wiegold; Generators and killers for direct and free products. Arch. Math. (Basel), 34(4) (1980) 296-300.

[4] J. Peterson and A. Thom; Group cocycles and the ring of affiliated operators. Invent. math., 185, Issue 3 (2011) 561-592. 
Nicolas Monod, EPFL, 1015 Lausanne, Switzerland

E-mail address: nicolas.monod@epfl.ch

Narutaka OzaWA, RIMS, Kyoto University, 606-8502, JAPAN

E-mail address: narutaka@kurims.kyoto-u.ac.jp

Andreas Thom, Univ. Leipzig, PF 100920, 04009 Leipzig , Germany

E-mail address: andreas.thom@math.uni-leipzig.de 